\title{
EchoGéo
}

$51 \mid 2020$

Territoires « cyclonés "

\section{Avis de tempêtes et temps de crises}

Éditorial

Jean-Louis Chaléard

\section{CpenEdition}

Journals

Édition électronique

URL : https://journals.openedition.org/echogeo/18616

DOI : 10.4000/echogeo.18616

ISSN : 1963-1197

Éditeur

Pôle de recherche pour l'organisation et la diffusion de l'information géographique (CNRS UMR 8586)

Référence électronique

Jean-Louis Chaléard, «Avis de tempêtes et temps de crises », EchoGéo [En ligne], 51 | 2020, mis en ligne le 25 avril 2020, consulté le 10 août 2021. URL : http://journals.openedition.org/echogeo/18616 DOI : https://doi.org/10.4000/echogeo.18616

Ce document a été généré automatiquement le 10 août 2021.

EchoGéo est mis à disposition selon les termes de la licence Creative Commons Attribution - Pas d'Utilisation Commerciale - Pas de Modification 4.0 International (CC BY-NC-ND) 


\title{
Avis de tempêtes et temps de crises
}

\section{Éditorial}

\author{
Jean-Louis Chaléard
}

1 Le numéro 51 d'échogéo sort dans un contexte difficile. La crise sociale qui secoue la France depuis plusieurs mois a (momentanément?) laissé place à la crise sanitaire liée à la pandémie du covid-19, d'une ampleur inégalée. Notre revue s'est clairement inscrite dans le mouvement de luttes sociales à propos des retraites et des projets de loi sur l'enseignement supérieur et la recherche. Mais, comme indiqué dans la motion présente sur notre site, nous n'avons pas voulu interrompre notre publication qui aurait surtout pénalisé les auteurs et autrices qui s'étaient déjà engagés dans la production d'articles et dans la réponse à des dossiers thématiques. Les mesures de confinement n'ont pas facilité la tâche des contributeurs à cette livraison ni celle du secrétariat de rédaction, qu'il faut remercier pour leurs efforts. Sans doute aurionsnous aimé avoir d'autres textes qui viendront alimenter les rubriques, espérons-le, dans les numéros à venir, les circonstances n'ayant permis de les mener à termes avant la parution de la présente livraison.

2 Le dossier principal, dans la rubrique Sur le champ, porte sur les « territoires cyclonés». Il serait facile de faire un rapprochement entre les tempêtes sociales, l'ouragan provoqué par le coronavirus et les cyclones tropicaux analysés dans le dossier. Mais les cataclysmes ne sont pas du même ordre. Contentons-nous d'observer qu'un certain nombre d'articles sur les cyclones apportent une réflexion sur la gestion des risques, leur anticipation, qui font écho aux questions soulevées dans les milieux scientifiques et les médias sur la crise du coronavirus (comme le texte de V. November et al. sur « le couple anticipation/décision aux prises avec l'exceptionnel, l'imprévu et l'incertitude », celui de F. Benitez et al. sur « la culture du risque cyclonique... », etc.). L'inégale vulnérabilité des populations renvoie clairement aux inégalités sociales dont souffrent les populations affectées, que ce soit dans l'Outre-mer français ou les pays étrangers. Mais le thème du dossier, lancé voici plus d'un an, dépasse largement les cadres de l'actualité, avec la réflexion qu'autorise le recul.

3 F. Leone et F. Vinet ont ainsi piloté un ensemble fourni de treize contributions proposant une approche géographique des phénomènes cycloniques, comme ils 
l'expliquent dans leur introduction. Celles-ci portent majoritairement sur les Antilles, particulièrement sur Saint-Martin, victime de l'ouragan Irma en 2017 et dont la presse a beaucoup parlé. Mais les articles abordant Madagascar (T. Catry et al.), ou le golfe d'Aden (J.-P. Cherel et al.) élargissent le champ d'étude et permettent d'enrichir les conclusions que l'on peut tirer. De même, si la plupart des communications sont centrées sur les cyclones les plus récents, certaines s'inscrivent dans le temps long, comme celle de F. Leone et al. qui analyse les relations mortalité-cyclones sur un pas de temps multiséculaire (1635-2018), ou reviennent sur des événement anciens comme celle de J. Desharte qui s'intéresse aux effets et à la gestion du cyclone de septembre 1928 en Guadeloupe, ou s'interrogent sur l'évolution des événements sur plusieurs décennies comme celle de K. Hoarau et al. qui prennent en compte la période 1945-2018. Les comparaisons peuvent ainsi opérer non pas seulement dans l'espace mais aussi dans le temps.

Un volet important est consacré aux aspects méthodologiques de l'étude des cyclones. L'approfondissement des réflexions et l'ouverture à des interrogations inédites vont, de ce point de vue, de pair avec le recours à de nouveaux outils, notamment dans le domaine de la télédétection et l'utilisation des images satellites. C'est particulièrement vrai dans le cas de l'article de T. Catry et al. sur « les apports de l'imagerie satellite pour le suivi de l'impact des événements cycloniques à Madagascar» qui propose une évaluation des zones inondées par les cyclones Haruna en 2013 et Enawo en 2017 dans la Grande île. De manière différente, des textes sur la connaissance des cyclones, sur les risques et leur gestion ont une approche qualitative, fondée sur des entretiens, l'élaboration de cartes par les enquêtés, etc. Les différentes méthodes ne s'excluent pas et des auteurs emploient des techniques différentes (comme T. Rey et al. qui ont recours aux images des satellites Pléiades, à des photographies des côtes, à des témoignages des personnes vivant sur les espaces affectés pour analyser les changements côtiers et les inondations suite au passage d'Irma). L'utilisation d'archives et/ou de bases de données conjointement avec des interviews d'acteurs et de témoins se retrouve dans plusieurs communications. F. Leone et al., particulièrement, posent les bases méthodologiques d'un catalogue historique des événements hydro-météorologiques qui ont frappé l'archipel de Guadeloupe et les îles du Nord ; l'étude repose sur l'utilisation de sources multiples (archives, coupures de presse, bases de données, etc.) et l'élaboration d'un SIG pour la période récente.

5 Les contributions offrent un large éventail des questions scientifiques et pratiques posées par les cyclones. L'origine des événements et leur fréquence sont abordées. Ainsi des auteurs s'interrogent-ils sur le rôle du réchauffement climatique dans l'évolution du nombre et de la violence des cyclones, que ce soit dans les Antilles (K. Hoarau et al.) ou en mer Rouge (J.-P. Cherel et al.), apportant dans les eux cas des conclusions nuancées et insistant sur la complexité des causalités. Les effets sur le milieu naturel sont mis en évidence. T. Rey et al., étudiant les changements côtiers et les inondations suite au passage d'Irma, soulignent aussi que les systèmes côtiers ont répondu très différemment en fonction du degré d'artificialisation du littoral.

Beaucoup d'articles abordent l'ampleur des dégâts occasionnés par les cyclones et le nombre de victimes. L'utilisation des nouvelles techniques permet de préciser les effets, comme on le voit à travers les communications de T. Catry et al. ou de T. Rey et al. Le bilan matériel et humain est souvent lourd, même dans le cas de cyclones moins violents mais ou l'impréparation en raison de la nouveauté du phénomène dans une 
région aride a des effets catastrophiques (J.-P. Cherel et al.). F. Leone et al. constatent, sur le temps long, que les décès sont de moins en moins liés à l'occurrence d'un cyclone, mais qu'ils sont de plus en plus associés à des comportements de mise en danger lors d'événements de faible ou moyenne intensité, conduisant à s'interroger sur la préparation des populations aux risques.

7 Au-delà du bilan matériel et humain, les textes proposent une réflexion sur la gestion de la crise, la vulnérabilité des populations et les formes de résilience, s'attachant à des populations ou des institutions particulières. V. Novembrer et al. s'intéressent au couple anticipation/décision en situation de crise, à travers deux études de cas, soulignant la fragmentation de ce couple, au sein, et entre, les cellules de crise et l'absence de vision partagée. S. Defossez et M. Gherardi s'interrogent sur le rôle des populations dans la gestion de la catastrophe à Saint-Martin. Les populations paraissaient bien préparées, mais à partir d'une référence ancienne qu'Irma a dépassé en intensité. Les dommages ont ainsi révélé ou exacerbé des vulnérabilités existantes qui questionnent les processus de reconstruction.

Plusieurs communications s'interrogent plus particulièrement sur les problématiques post-crises. A. Moatty et al. abordent la question du relèvement après le cyclone Irma à Saint-Martin à partir de l'analyse du vécu des adolescents et tentent de caractériser les actions qu'ils ont menées. M. Cherchelay, s'intéressant au tourisme, activité majeure à Saint-Martin, qui a fortement souffert du passage d'Irma, met en évidence les jeux d'acteurs complexes qui se développent autour des enjeux et des attentes qui émergent en période de reconstruction. L'aide internationale est évoquée dans de nombreux cas. Elle est au centre de la contribution de S. Veitl qui pose un regard critique sur cette assistance à propos du cas de la reconstruction des écoles dans le département du sud d'Haïti après l'ouragan Matthew de 2016, mettant en évidence la volonté des organismes humanitaires qui veulent insuffler un nouveau modèle de développement et les résistances de la réalité aux injonctions d'adaptation.

Enfin des articles, au-delà des effets immédiats, abordent les évolutions économiques et sociales ou les adaptations face au risque cyclonique à plus long terme. L'étude de l'ouragan de septembre 1928 en Guadeloupe par J. Desarthe met en évidence les conséquences directes (mortalité, destructions) et indirectes, notamment sur la filière sucrière qui a reçu une intervention massive de l'État, sur la culture de la banane en essor et sur des productions anciennes comme le café et le cacao, en déclin. F. Benitez et al. s'interrogent sur la notion de "culture de risque» par les différents acteurs du champ de la prévention des catastrophes naturelles et de la gestion de crise. Les auteurs montrent que les individus ont conscience du risque cyclonique mais que le danger est souvent sous-évalué et la préparation inégale, en raison de situations variées à prendre en compte. M. Desse et al. replacent les activités et les sociétés dans un cadre plus large et mettent en évidence la complexité des évolutions à la Guadeloupe à partir de l'examen des cyclones Hugo de 1989 et Dean de 2007. Les cyclones frappent des économies insulaires très dépendantes de l'activité touristique et des littoraux, souvent en crise, et leurs conséquences sont variables selon les contextes économiques, les espaces étudiés et les acteurs concernés. Comme la plupart des auteurs qui ont participé au dossier, leurs réflexions s'inscrivent dans des débats plus généraux sur les risques et sur la diversité des effets à prendre en compte lors des catastrophes.

Dans un tout autre ordre d'idées, G. Gillot et A. Sierra, dans la rubrique Sur le métier, ont interviewé quatre collègues géographes, enseignants et/ou chercheurs, sur la future 
LPPR (loi de programmation pluriannuelle de la recherche) et les mouvements qui ont touché le monde universitaire et de la recherche fin 2019- début 2020. Il s'agit de M. Blidon (Université Paris 1 Panthéon-Sorbonne), J.-B. Frétigny (Cergy Paris Université), C. Luxembourg (École nationale supérieure d'architecture de Paris-La Villette) et J. Brachet (IRD). Au-delà des opinions propres à chacun, le dossier est l'occasion de revenir sur la politique générale de l'enseignement supérieur et de la recherche menée depuis de nombreuses années en France et dans laquelle s'inscrivent les projets de réforme à venir. C'est l'occasion aussi pour les intervenants de proposer leurs analyses des causes et des modalités de la contestation récente. Ils soulignent l'importance des mobilisations en sciences sociales et humaines, ne singularisant pas, de ce point de vue, la géographie, mais l'inscrivant dans un ensemble plus large. La réflexion débouche sur une réflexion sur la pandémie du coronavirus, et sur ses conséquences sur la recherche. On revient alors à des questions de fonds sur la prévention des risques, le rôle de la recherche dans ce contexte et les enseignements à tirer des crises actuelles. 